declared, Rossana Scrivo: None declared, Carlo Perricone: None declared, Guido Valesini: None declared, fabrizio conti Speakers bureau: BMS, Lilly, Abbvie, Pfizer, Sanofi

DOI: 10.1136/annrheumdis-2020-eular.5987

\section{SAT0556 FINE STRUCTURE ANALYSIS OF THE INTER- RELATION BETWEEN TOPHUS DEPOSITION AND BONE LESIONS IN GOUT USING A COMBINATION OF DUAL ENERGY AND HIGH-RESOLUTION CT}

S. Bayat ${ }^{1}$, D. Simon ${ }^{1}$, C. Pecherstorfer ${ }^{1}$, H. Ellmann ${ }^{1}$, C. Figueiredo ${ }^{2}$, M. Englbrecht ${ }^{1}$, A. Hueber ${ }^{3}$, A. Kleyer ${ }^{1}$, J. Rech ${ }^{1}$, G. Schett ${ }^{1} .{ }^{1}$ Department of Internal Medicine 3 - Rheumatology and Immunology, Friedrich-Alexander University (FAU) Erlangen-Nürnberg and Universitätsklinikum Erlangen, Erlangen, Germany, Erlangen, Germany; ${ }^{2}$ Bone Metabolism Laboratory, Rheumatology Division, Faculdade de Medicina da Universidade de São Paulo, São Paulo, Brazil; ${ }^{3}$ Section Rheumatology, Sozialstiftung Bamberg, Bamberg, Germany

Background: Deposition of uric acid crystals cause an inflammatory reaction, which can lead to structural bone changes, if such deposits form adjacent to cortical bone [1, 2]. Both erosions and bony spurs can form in conjunction with tophus deposition. The exact spatial inter-relation between tophi and structural bone lesions in humans in vivo is not fully characterized.

Objectives: To spatially relate structural bone changes (erosions, osteophytes) to the deposition of monosodium urate crystals in the first metatarsophalangeal (MTP1) joint in patients with tophaceous gout.

Methods: Tophaceous gout patients with clinically detected tophi at the MTP1 joint underwent simultaneous dual energy computed tomography (DECT) and high-resolution peripheral quantitative computed tomography (HR-pQCT) of the feet. Tophi detected by DECT and erosions and osteophytes detected by HR-pQCT were overlayed to define their exact anatomical relation. Furthermore, feet of sex- and age-matched healthy controls $(\mathrm{HC})$ were scanned to define the normal architecture of the MTP1 joint.

Results: Gout patients $(\mathrm{N}=20)$ had significantly higher numbers (5 (0-17 vs. 1 $(1-2))$ and volumes $\left(45.32 \mathrm{~mm}^{3}(7.26-550.32)\right.$ vs. $\left.0.82 \mathrm{~mm}^{3}(0.15-21.8)\right)$ of bone erosions as well as significantly higher numbers (10.5 (0-26) vs. $1(0-10))$ and sizes of osteophytes $(4.93 \mathrm{~mm}(0.77-7.19 \mathrm{~mm}$ vs. $0.93 \mathrm{~mm}(0.05-7.61 \mathrm{~mm})$ than healthy controls $(\mathrm{N}=20)$. Erosions were in direct spatial relation to bone erosions, while osteophytic responses were more widespread and affected bone regions on the MTP1, which were not directly adjacent to tophi. Median tophus volume detected by DECT $\left(0.12 \mathrm{~mm}^{3}(0.01-2.53)\right)$ was associated with the total volume of erosions $(r=0.597, p=0.005)$.

Conclusion: This study demonstrates that bone changes in gout are substantial and not only include erosions but also widespread architectural bone remodeling associated osteophyte formation. While there is a direct spatial relation between tophi and bone erosions the anabolic bone responses in gout are more widespread.

References:

[1] Dalbeth, N. et al. Ann Rheum Dis. 2015 Jun;74(6):1030-6.

[2] Dalbeth, N. et al. Arthritis Res Ther. 2012; 14(4): R165.
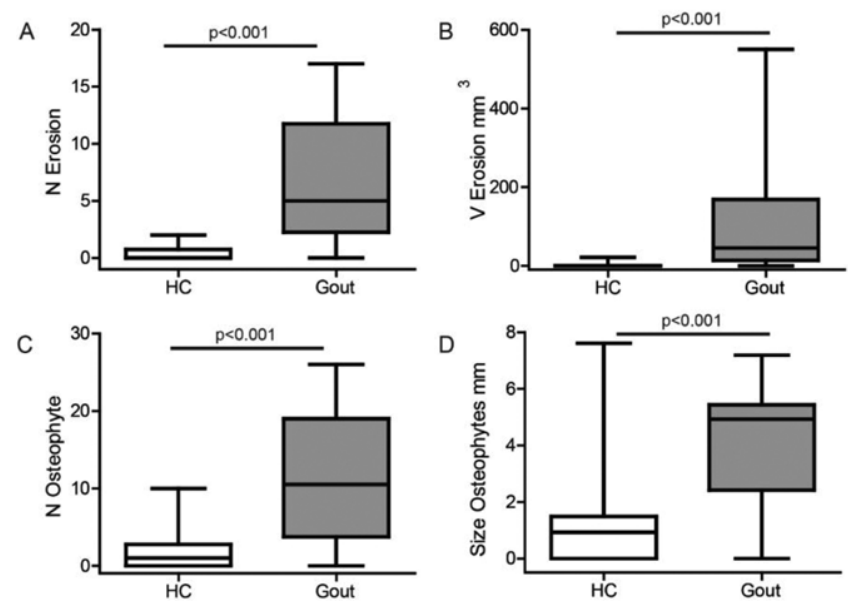

Data are based on high-resolution peripheral quantitative computed tomography (HR-pQCT) of metatarsophalangeal joints I in gout patients (grey boxplots) and healthy controls (white boxplots). (A) number of bone erosions, (B) volume of bone erosions, (C) number of osteophytes and (D) size of osteophytes. Data are shown as medians and inter-quartile ranges (boxes).

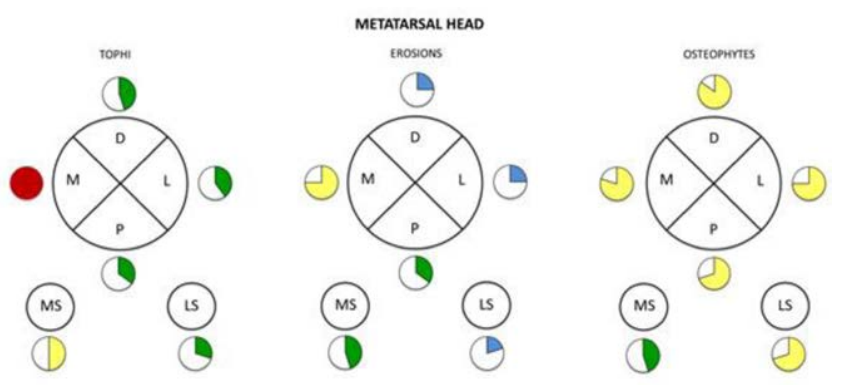

Distribution of $(A)$ tophi based on dual-energy computed tomography (DECT) as well as (B) bone erosions and (C) osteophytes based on high-resolution peripheral quantitative computed tomography (HR-pQCT) of metatarsophalangeal (MTP) I head in gout patients. Data are shown for the different regions of the MTPI head including the plantar, medial, dorsal and lateral region of the metatarsal head, as well as the medial and lateral sesamoid bones. Data indicate percentage of patients with tophi, erosions and osteophytes in respective region.

Disclosure of Interests: Sara Bayat Speakers bureau: Novartis, David Simon Grant/research support from: Else Kröner-Memorial Scholarship, Novartis, Consultant of: Novartis, Lilly, Caroline Pecherstorfer: None declared, Hanna Ellmann: None declared, Camille Figueiredo: None declared, Matthias EngIbrecht: None declared, Axel Hueber Grant/research support from: Novartis, Lilly, Pfizer, EIT Health, EU-IMI, DFG, Universität Erlangen (EFI), Consultant of: Abbvie, BMS, Celgene, Gilead, GSK, Lilly, Novartis, Speakers bureau: GSK, Lilly, Novartis, Arnd Kleyer Consultant of: Lilly, Gilead, Novartis,Abbvie, Speakers bureau: Novartis, Lilly, Jürgen Rech Consultant of: BMS, Celgene, Novartis, Roche, Chugai, Speakers bureau: AbbVie, Biogen, BMS, Celgene, MSD, Novartis, Roche, Chugai, Pfizer, Lilly, Georg Schett Speakers bureau: AbbVie, BMS, Celgene, Janssen, Eli Lilly, Novartis, Roche and UCB DOI: 10.1136/annrheumdis-2020-eular.2151

\section{\begin{tabular}{|l|l}
\hline SAT0557 RESIDUAL DISEASE ACTIVITY IN ADULT-ONSET \\
\hline
\end{tabular} STILL'S DISEASE: QUALITATIVE AND QUANTITATIVE ANALYSIS OF A SERIES OF 18F FDG-PET/MR}

S. Bindoli ${ }^{1}$, P. Galozzi ${ }^{2}$, F. Magnani ${ }^{3}$, G. Abruzzino ${ }^{2}$, D. Cecchin ${ }^{3}$, P. Sfriso ${ }^{4}$. ${ }^{1}$ University of Padova, Rheumatology Unit, Padova, Italy; ${ }^{1}$ University of Padova, Rheumatology Unit, Padova, Italy; ${ }^{3}$ University of Padova, Nuclear Medicine Unit, Padova, Italy; ${ }^{1}$ University of Padova, Rheumatology Unit, Padova, Italy

Background: Adult-onset Still's disease (AOSD) is a systemic autoinflammatory disorder characterized by episodes of spiking fever, the presence of an evanescent pink-salmon rash, arthritis/arthralgias, sore throat and increased inflammatory serum markers. The diagnosis is clinical and needs the exclusion of potential mimickers such as infections and lymphoproliferative disorders. Currently, a specific diagnostic test to assess the disease activity is not available.

Objectives: To define the residual disease activity in AOSD and establish a possible response to therapy through ${ }^{18} \mathrm{~F}$-FDG PET/MR imaging technique.

Methods: 23 patients affected by AOSD and 24 controls underwent ${ }^{18} \mathrm{~F}$-FDG PET/MR between 2014 and 2018. A total of $54{ }^{18} \mathrm{~F}$-FDG PET/MR were analysed. AOSD patients were diagnosed according to the Yamaguchi's criteria and were in follow-up at the Rheumatology Unit of Padova University Hospital. The controls were chosen among non-AOSD patients with a previous diagnosis of solid tumors (lymphomas excluded). A qualitative analysis of PET/RM carried out by a Nuclear Medicine Specialist and a semiquantitative analysis carried out by measuring SUVs-to-liver (Standardized Uptake Value) for spleen, bone marrow (BM), lymph nodes and pharynx were performed. A SUVmax BM/SUVmean liver higher than 2.09 was set up as significant area of uptake for each organ considered. This threshold was calculated by adding the standard deviation multiplied by 2 at the mean ratio between SUVmax BM and SUVmean liver of the control group. The Pouchot score for disease activity was calculated for each subject. The distribution of the variables was investigated by Shapiro-Wilk test. The analysis of the association between the variables was carried out using the Mann-Whitney U test.

Results: AOSD patients present areas of focal ${ }^{18} \mathrm{~F}-\mathrm{FDG}$ uptake mainly in BM, lymph nodes, pharynx, spleen and salivary glands. Sites of uptake in spleen were found in $3.3 \%$ of PET/MR, in BM in $23.3 \%$, in lymph nodes in $23.3 \%$ and in pharynx in $36.6 \%$ of PET/RM respectively. Eleven/thirty (47.8\%) patients were 